POS $\quad \begin{aligned} & \text { PROCEEDINGS } \\ & \text { OF SCIENCE }\end{aligned}$

\title{
Decay studies at the end of the rp-process
}

\author{
E.E. Smith", M. E. Howard, B. Mercurio, S. D. Reitzner, \\ Department of Physics, The Ohio State University \\ Columbus, $\mathrm{OH}$ 43210, USA \\ E-mail: edesmith@mps .ohio-state.edu
}

\section{A. Estrade, P. T. Hosmer, E. Kwan, S. N. Liddick, P. F. Mantica, F. Montes, A. C. Morton, H. Schatz, A. Stolz, B. E. Tomlin \\ National Superconducting Cyclotron Lab, Michigan State University \\ East Lansing, MI 48824, USA}

The rapid proton-capture process (rp-process) produces proton rich nuclei on the surface of accreting neutron stars. The light curves of type I X-ray bursts are an observable result of the energy produced. In reaction networks, a series of (p, $\gamma$ ) reactions and $\beta$-decays proceed along a path close to the proton drip-line culminating, for some bursts, in a $\mathrm{Sn}-\mathrm{Sb}$ - Te cycle. To address uncertainties in the masses near the proton drip-line, we have observed the $\beta$-decay of neutron-deficient ${ }^{103,104,105} \mathrm{Sb}$ isotopes at the National Superconducting Cyclotron Laboratory at Michigan State University. A mixed beam of unstable nuclei was produced at the Coupled Cyclotron Facility and isolated in the A1900 fragment separator. Nuclei were then implanted and decays were observed in the NSCL $\beta$-decay end station. We discuss preliminary results on $\beta$-decay properties and the search for proton radioactivity.

International Symposium on Nuclear Astrophysics - Nuclei in the Cosmos - IX

CERN, Geneva, Switzerland

25-30 June, 2006

\footnotetext{
${ }^{*}$ Speaker
} 


\section{Introduction}

The rapid proton capture process (rp-process) on the surface of accreting neutron stars fuses hydrogen into heavier elements supplying the energy that leads to type I X-ray bursts. The endpoint for an individual burst may depend upon conditions such as the peak temperature and the amount of available $\mathrm{H}$ for ignition. Reaction network calculations show that the natural limit of the rp-process occurs due to a $\mathrm{Sn}-\mathrm{Sb}$ - Te cycle [1]. When reaction flows reach the range of light Sn nuclei near doubly magic ${ }^{100} \mathrm{Sn}, \beta$-decay of Sn nuclei and proton capture by In nuclei allow the reactions to follow a path along the chain of $S n$ isotopes towards greater $\beta$ stability. The reaction flow's primary path passes from ${ }^{105} \mathrm{Sn}$ to ${ }^{106} \mathrm{Sb}$ to ${ }^{107} \mathrm{Te}$ along the first set of isotones that are sufficiently proton-bound to allow $(p, \gamma)$ proton capture to dominate the rate of $(\gamma, \mathrm{p})$ photodisintegration. Upon reaching ${ }^{107} \mathrm{Te}$, however, $(\gamma, \alpha)$ photodisintegration produces ${ }^{103} \mathrm{Sn}$ once again, creating the aforementioned cycle.

The $\mathrm{Sn}-\mathrm{Sb}$ - Te cycle has consequences for the composition of the resulting ashes. First, it imposes an upper mass limit near $\mathrm{A}=107$. Second, it allows for the production of a broad range of nuclei in the $\mathrm{A}=64-107$ mass region. Furthermore, the cycle allows for the build-up of the relatively long-lived ${ }^{104} \mathrm{Sn}$, alters the relative abundances of hydrogen and helium, and increases energy production at late times in the burst. These factors will observably alter the Xray burst, lengthening the tail. The resulting abundances of p-nuclei, such as ${ }^{92,94} \mathrm{Mo}$ and ${ }^{96,98} \mathrm{Ru}$, depend upon both the cycle itself and the ability of the ash to escape the neutron star [1].

While it is clear that the $\mathrm{Sn}-\mathrm{Sb}-\mathrm{Te}$ cycle forms once the rp-process reaches the neutron deficient Te isotopes, it is still uncertain at which $\mathrm{Sn}$ isotope the proton-capture reaction flow breaks through the weakly bound Sb isotopic chain and whether there are significant secondary branches. The neutron deficient ${ }^{106-108}$ Te are experimentally known ground state alpha emitters and are alpha unbound by 3-4 MeV [2,3]. Decay properties and proton separation energies among the antimony isotopes, however, are less certain. These values play roles in determining the degree to which the cycle follows the primary isotonic chain $\left({ }^{105} \mathrm{Sn}-{ }^{106} \mathrm{Sb}-{ }^{107} \mathrm{Te}\right)$ or a possible sub-cycle (starting with e.g. ${ }^{106} \mathrm{Sn}-{ }^{107} \mathrm{Sb}-{ }^{108} \mathrm{Te}$ or ${ }^{104} \mathrm{Sn}-{ }^{105} \mathrm{Sb}-{ }^{107} \mathrm{Te}$ ) [1].

The neutron deficient antimony isotopes observed in the present experiment, ${ }^{103,104,105} \mathrm{Sb}$, have all been previously observed and in each case, some decay property uncertainties have remained. The first reports of ground-state proton decay of ${ }^{105} \mathrm{Sb}$ were made in 1994 by a Berkeley group [4]; they observed a $478 \pm 15 \mathrm{keV}$ line to be a ground state proton decay and assigned this decay mode a branching ratio of $1 \%$. However, this result could not be confirmed in subsequent studies [5-8], though the detection of a $550 \pm 30 \mathrm{keV}$ proton with a similar branching ratio has been reported by one group [5]. Most recently, Liu et. al. have presented data suggesting that for proton energies greater than $430 \mathrm{keV}$, the upper limit of the ground state proton decay branching ratio is $\sim 10^{-3}$ [6]. In a previous fragment separator study at GSI, the

${ }^{105} \mathrm{Sb}$ half-life was measured to be $\sim 1.12 \mathrm{~s}$ [5]. The nuclei ${ }^{103,104} \mathrm{Sb}$ were first observed at GANIL [7]. The ${ }^{104} \mathrm{Sb}$ half-life has been measured in a GSI experiment to be $0.44_{-0.11}^{+0.15} \mathrm{~s}[6]$. 


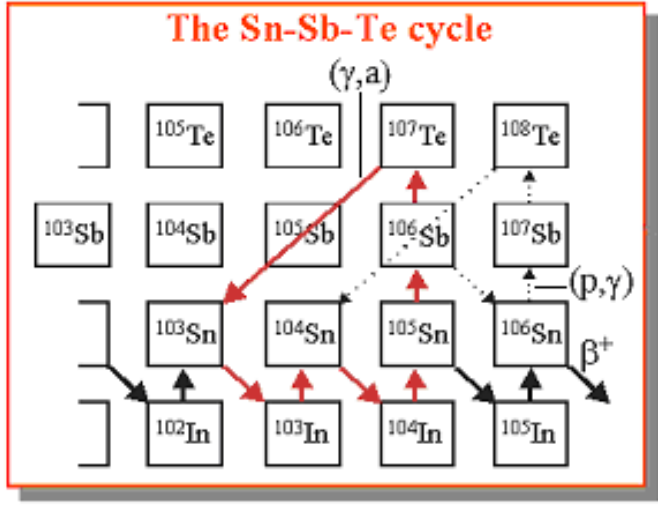

FIGURE 1: Reactions within the $\mathrm{Sn}-\mathrm{Sb}-\mathrm{Te}$ cycle. The upward arrows represent points where the proton capture rate is thought to exceed the reverse flow due to $(\gamma, \mathrm{p})$ photodisintegration. Red arrows show the primary $\mathrm{Sn}-\mathrm{Sb}$ - Te cycle. Dashed lines show one possible secondary pathway (from [1]).

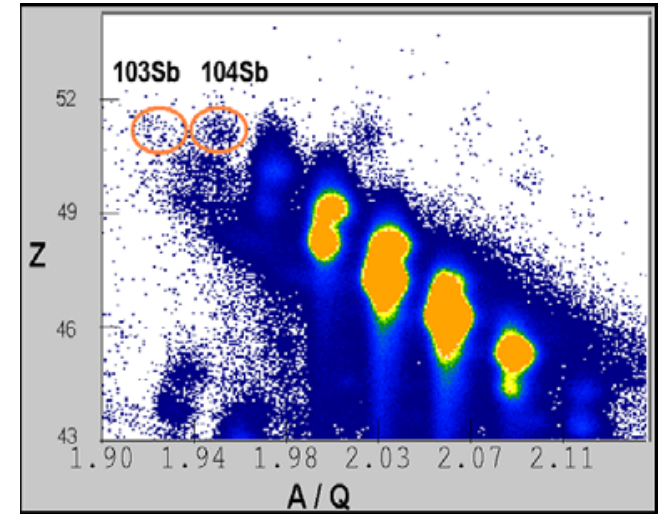

FIGURE 2: Particle ID (atomic number versus mass/charge ratio). Color indicates the number of counts on a logarithmic scale.

Proton deficient antimony isotopes also have relevance for nuclear structure studies [8]. The lightest isotope observed to date, ${ }^{103} \mathrm{Sb}$ for example, has just one proton and two neutrons more than doubly magic ${ }^{100} \mathrm{Sn}$ and thus lies simultaneously at the extremes of stability and close to a closed nuclear shell. The goal of the present experiment is to measure the $\beta$-decay half-life of ${ }^{103} \mathrm{Sb}$, to re-measure the half-lives of ${ }^{104,105} \mathrm{Sb}$ and to search for proton emission from these isotopes.

\section{The $\beta$-decay experiment at the NSCL}

${ }^{103,104,105} \mathrm{Sb}$ isotopes were produced at the National Superconducting Cyclotron Laboratory. A $120 \mathrm{MeV} / \mathrm{A}$ primary beam of ${ }^{124} \mathrm{Xe}$ was provided by the Coupled Cyclotron Facility and collided with a ${ }^{9} \mathrm{Be}$ target. The resulting mixed fragment beam was separated in the A1900 fragment separator [9] and nuclei were implanted into a 40 by 40 strip (1600 pixel) Doublesided Silicon Strip Detector (DSSD) at the center of the NSCL $\beta$-decay calorimeter $[10,11]$. The calorimeter also includes a pair of energy loss detectors to observe the incoming particles and a stack of nine additional detectors to further characterize events. NSCL segmented germanium detectors [12] were used to observe $\gamma$-rays from isomeric states of implanted nuclei and $\beta$-delayed $\gamma$-rays.

\section{Results}

\subsection{Particle Identification}

Implanted nuclei can be characterized by energy loss $(\Delta \mathrm{E})$ and time-of-flight (TOF). For fully stripped nuclei it is possible to calculate atomic number $(Z)$ and mass/charge ratio $(A / Q)$, allowing us to identify nuclei event by event. Figure 2 shows the particle ID spectrum for the experiment. Figure 3 shows the $\mathrm{Z}$ distribution of implanted ions, where a correlated $\beta$-decay 
has been detected, cut into groups with similar values of A/Q. All plots are compilations from various fragment settings used over the duration of the experiment. The identity of particles in these plots has been confirmed through the observation of several gamma energies associated with the decay of a known $60 \mathrm{~ns}$ isomer in ${ }^{100} \mathrm{Cd}$.
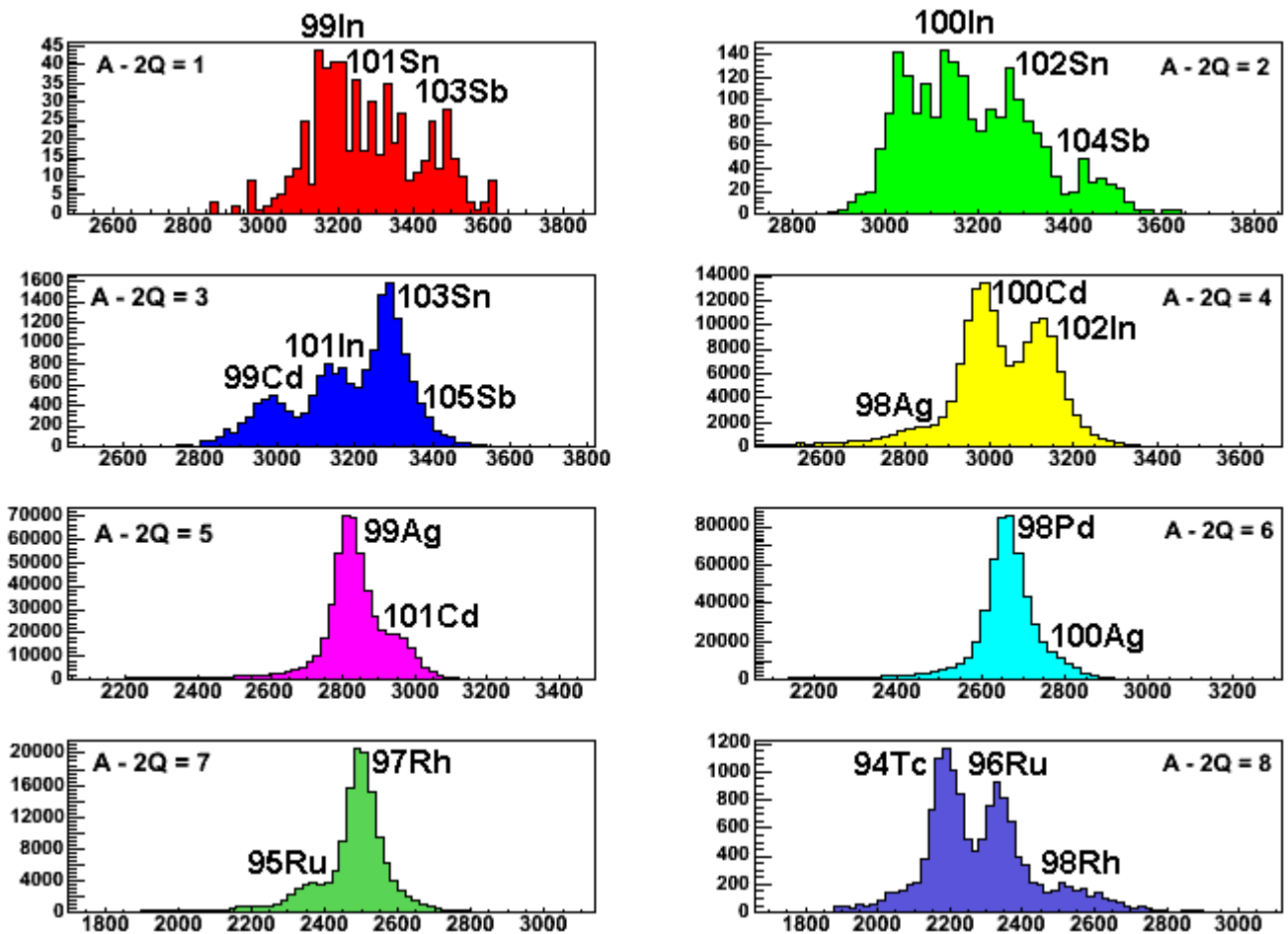

FIGURE 3: Z distribution of observed nuclei grouped according to the value of $\mathrm{A}-2 \mathrm{Q} .{ }^{97} \mathrm{Rh},{ }^{98} \mathrm{Pd},{ }^{99} \mathrm{Ag}$, and ${ }^{100,101} \mathrm{Cd}$, are all implanted into the detectors at a rate 3 or more orders of magnitude greater than that of the ${ }^{103,104} \mathrm{Sb}$ rate. These result in a high rate of background $\beta$ activity and complicate event correlation.

\section{2 $\beta$-decay half-life life and the search for proton activity}

The high rate of undesirable nuclei within the beam presents a number of challenges. Regions of the DSSD receiving the highest intensity of the beam have nuclei implanted at an average rate of 1 every 10 to 20 seconds per pixel. The majority of these nuclei have half-lives that range from 1 - 30 minutes. Decays from these species create a substantial and persistent background of decay events unrelated to the nuclei of interest. Also, since nuclei are most easily correlated only to the most recent implant, correlation windows for data collection may be cut short when an additional implant interrupts.

As these effects lead to a time dependent background for decay events following an implantation, the challenge is to accurately characterize this background. One approach is the development of a function that predicts the background spectra given the number of nuclei implanted. The background appears to be fit well by an exponential curve that scales linearly to the number of nuclei implanted for each gated nucleus. This function can then be incorporated into a maximum-likelihood-fit (preliminary fits are shown in Figure 4). 

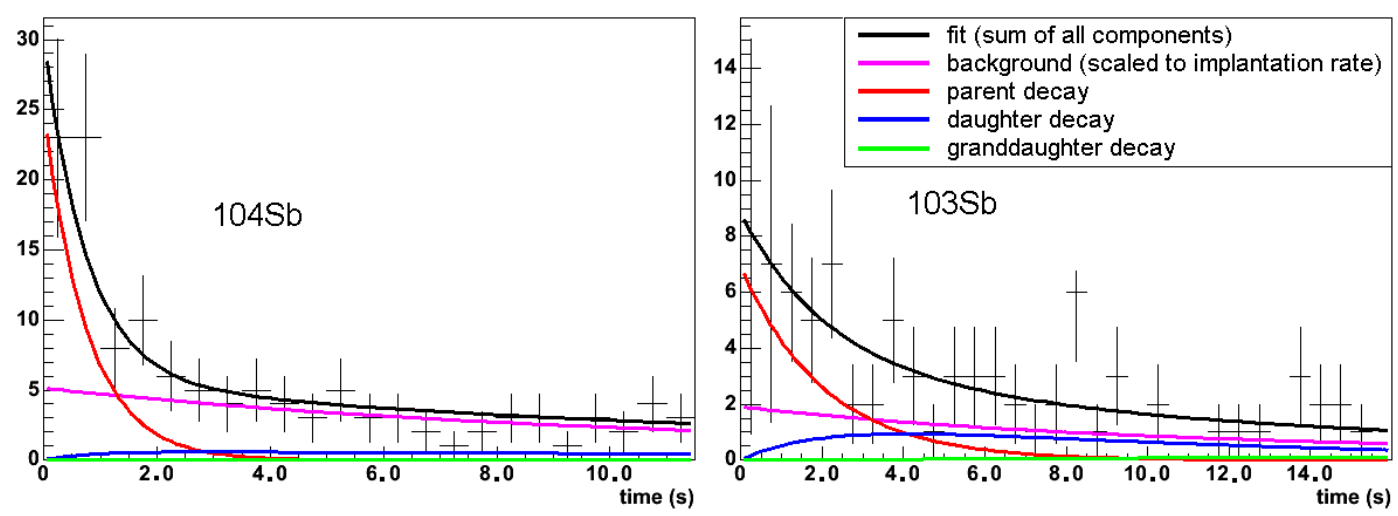

FIGURE 4: Preliminary fits of decay curves, ${ }^{104} \mathrm{Sb}$ left and ${ }^{103} \mathrm{Sb}$ right. This display has been binned and scaled for visualization. The fit is achieved with a maximum-likelihood-fit to the data in $10 \mathrm{~ms}$ bins. The preliminary results for ${ }^{104} \mathrm{Sb}$ are in agreement with the previous measurement.

Preliminary results in the search for proton activity are inconclusive due to the high rate of background events. We defined "proton-like" events to be those that occurred within five seconds of the nucleus being implanted, occurred in a single channel of both the front and back of the DSSD, and had good agreement between the front and back energies.

\section{Summary and outlook}

The culmination of the rapid proton-capture process into a $\mathrm{Sn}-\mathrm{Sb}-\mathrm{Te}$ cycle has implications for the luminosity curves associated with X-ray bursts from accreting neutron stars and places clear limits upon the nucleosynthesis that can occur within these systems. The decay properties of neutron deficient antimony help to constrain the path of reactions that occur in this cycle. ${ }^{103,104,105} \mathrm{Sb}$ have been observed in the NSCL $\beta$-decay calorimeter. Although the background rate is high, the half-lives of ${ }^{103,104} \mathrm{Sb}$ are expected to be relatively short compared to the time between implantations. Correlations may be possible as is exhibited in Figure 4, where a definite short-lived decay component is observed for $\beta$ events correlated with ${ }^{103,104} \mathrm{Sb}$ implantations. Continued analysis of these data may permit first determination of the ${ }^{103} \mathrm{Sb}$ halflife.

The lack of beam purity observed in this experiment is a common problem among rare isotope beams of neutron deficient nuclei at NSCL energies. Often, high intensities of unwanted nuclei are present in the mixed secondary beam. When such nuclei have similar magnetic rigidities and energy losses in matter as the experimentally desirable nuclei, they cannot easily be separated in the NSCL A1900 fragment separator.

In the near future, a solution to the problem of contaminants in neutron-deficient beams will be achieved with the installation of a radio frequency fragment separator (RFFS) [13]. The RFFS will filter beam impurities based on their differing velocities, which will translate into a phase difference with respect to the cyclotron RF. Simulations indicate a possible background reduction of several orders of magnitude [14]. 


\section{Acknowledgements}

Funding for this research has been provided by the NSF through grants PHY-0140255, PHY0110253, and PHY-0216783 (The Joint Institute for Nuclear Astrophysics).

\section{References}

[1] H. Schatz et. al., End Point of the rp Process on Accreting Neutron Stars, Phys. Rev. Lett. 86:3471 (2001).

[2] D. Schardt, et. al., Alpha decay studies of tellurium, iodine, xenon, and cesium isotopes, Nucl. Phys. A 326:65 (1979).

[3] R.D. Page, Alpha radioactivity above ${ }^{100}$ Sn including the decay of ${ }^{108} I$, Phys. Rev. C 49:3312 (1994).

[4] R. J. Tighe et. al., Evidence for the ground-state proton decay of ${ }^{105} \mathrm{Sb}$, Phys. Rev. C. 49:R2871 (1994).

[5] J. Friese, in Proceedings for the International Workshop XXIV on Gross Properties of Nuclei and Nuclear Excitations, Hirschegg, Austria, 1996, edited by H. Feldmeier, J. Knoll, and W. Norenberg (GSI, Darmstadt, 1996) p.123; Preliminary results also presented in the following: R. Schneider, Physica Scripta T56:67 (1995); R. Schneider, Nucl. Phys. A588:191c (1995).

[6] Z. Liu, et. al., Reinvestigation of direct proton decay of ${ }^{105} S b$, Phys. Rev. C. 72:047301 (2005).

[7] M. Lewitowicz, Identification of ${ }^{100}$ Sn and Other Proton Drip-Line Nucleiin the Reaction ${ }^{112} \mathrm{Sn}(63 \mathrm{MeV} / n u c l)+$. nat Ni, Nucl. Phys. A 588:197c (1995); see also: K. Rykaczewshki, Identification of new nuclei at and beyond the proton drip line near the doubly magic nucleus ${ }^{100} \mathrm{Sn}$ Phys. Rev. C. 52:2310 (1995).

[8] M. Shibata et. al., Beta-decay studies of ${ }^{107}$ Sb and other neutron-deficient antimony isotopes, Phys. Rev. C 55:1715 (1997).

[9] D.J. Morrissey, Commissioning the A1900 projectile fragment separator, Nucl. Instr. Meth. B 204:90, (2003).

[10] J.I. Prisciandaro, Beta counting system for fast fragmentation beams, Nucl. Instr. Meth. A 505:140 (2003).

[11] A.C. Morton, Beta-decay studies at the NSCL using a double-sided silicon strip detector, AIP Conference Proceedings, 680:550 (2003).

[12] W.F. Mueller et. al., Thirty-two-fold segmented germanium detectors to identify $\gamma$-rays from intermediate-energy exotic beams, Nucl. Instr. Meth. A 466:492 (2001)

[13] D. Gorelov et. al., rf-kicker system for the secondary beams at the NSCL. Proceedings of 2005 Particle Accelerator Conference, Knoxville, Tennessee.

[14] D. Bazin et. al., The Program LISE: a simulation of fragment separators, Nucl. Inst. Meth. A 482:307 (2002). 